\title{
Optimised mode selection in electromagnetic sensors for real time, continuous and in- situ monitoring of water cut in multi-phase flow systems
}

\author{
Chao Yuan ${ }^{1,2}$, Alexander Bowler ${ }^{2}$, Jonathan Gareth Davies ${ }^{2}$, Buddhika Hewakandamby ${ }^{2}$ and \\ Georgios Dimitrakis ${ }^{2 *}$
}

\author{
1 School of Electrical and Information Engineering, Tianjin University, Tianjin, 300072, China \\ 2 Faculty of Engineering, University of Nottingham, Nottingham, NG7 2RD, United Kingdom \\ *corresponding author email: georgios.dimitrakis@ nottingham.ac.uk
}

\begin{abstract}
Measurements of the composition of multiphase flows, especially water-cut in oilwater flow, is a frequently encountered problem in the petroleum industry. New techniques that can offer improvements towards that are continuously sought and the use of microwave sensors is considered very promising. The current paper reports on the performance of a microwave cylindrical resonator, integrated into a multiphase flow experimental facility, used to determine water-cut in an upward flowing oil-water mixture. The performance and suitability of two microwave resonant modes $\left(\mathrm{TM}_{010}\right.$ and $\left.\mathrm{TM}_{110}\right)$ was studied. The $\mathrm{TM}_{110}$ mode was found to be less dependent on the spatial phase distribution of the oil-water flowing mixture and provided more consistent results across a broader flow regime. The relative errors between the predicted and actual water-cut were from $-6.53 \%$ to $9.16 \%$ and relative errors of $78 \%$ of the total data points were inside $-5 \%$ to $5 \%$. The results suggest that appropriate sensor design and careful selection of the operating frequency/resonant pattern can offer a powerful technique for real time, on-line and non-destructive determination of water-cut in multiphase flow systems.
\end{abstract}

Key words: resonant cavity, oil-water flow, water volume fraction, resonant frequency, quality factor

\section{Introduction}

Accurate determination of water volume fraction (also known as water-cut) in oil-water mixtures under flow, which often encountered during oil production and transportation operations, is of great significance in the oil industry [1]. To date, many techniques have been proposed for real time measurements of water-cut in oil-water flowing mixtures, such as X-ray, gamma ray attenuation [2, 3], capacitance [4] and wire-mesh sensors [5]. However, there is a number of limitations associated with the uses of these techniques. X-ray and $\gamma$-ray based techniques require careful shielding and implementation as potentially they pose safety related risks. Such risks and also the mitigation steps that required to be implemented (shielding, access denial etc.) may render those techniques less attractive in an environment where space often is at a premium and there is constant presence of working personnel [6, 7]. Also $\gamma$-ray based techniques are known to be affected significantly by factors such as water salinity and the diameter of the pipes [8]. Wire-mesh sensors, being an invasive method, are interfering with the flow pattern and also are susceptible to oil corrosion, scaling and fouling which reduces the reliability of the measurements in the long term, and they are limited to flows in which the continuous phase is a conductive medium [6]. Capacitance sensors are suitable for mixtures of non-conductive continuous phase. The reconstruction calculations are not easy to 
perform and the spatial resolution remains poor [6]. Recent years have witnessed the investigation into the use alternative sensing techniques for the purpose of determining the composition of multiphase flow, one of the proposed techniques is based on the use of sensors utilising microwave frequencies [9].

Microwave based techniques can be non-intrusive, offering minimal disruption to flow pattern inside a pipe, which makes them ideal for the characterisation of flow systems. Using microwave sensors to measure the moisture of various materials, or including microwave sensors in advanced meters because of their extraordinary sensitivity to water, is one the most important groups of microwave sensing applications [10,11]. Consequently, the potential to carry out non-intrusive, online measurements of water-cut in oil-water flows, led to microwave sensors gradually attracting the attention of scientists and engineers and have been proposed in a number industrial applications for multiphase flow measurements [12]. Yang et al. [13] described an oscillator load-pull system to accurately determine the water-cut of an oil-water emulsion and presented the installation of the instrument and the field test results, but the watercut range was limited between $0-3 \%$. Avila et al. [14] determined water fraction from 0 to $100 \%$ by the frequency shift of the first resonant peak in a non-intrusive way based on a radio frequency resonant cavity sensor, however did not provide any details under flow conditions. Oon et al. [15] utilised the resonant frequencies occurring in a cylindrical cavity to monitor the changes in the permittivity of the measured phases to differentiate between the volume fractions of air, water and oil, however their work focused only on stratified flow. Ashton et al. [16] proposed a prototype non-intrusive microwave multiphase flow meter for measurement of oil, water and gas flow rates on production pipelines, focusing on large water-cut values ranging from $25.4 \%$ to $95.6 \%$. Hogan et al. [17] developed a real-time, non-intrusive multiphase dielectric meter capable of measuring the dielectric properties of different mixtures of oil, gas and water in full well stream flow, but this sensor could not be used in annular or churn flow. Wylie et al. [18] developed an electromagnetic cavity resonator based sensor, which was non-intrusive and transmitted low power $(10 \mathrm{~mW})$ radio frequencies in the range of 100-350 MHz, to detect the pipeline contents. The comparison between initial small scale tests and tests conducted at flow facilities in the National Engineering Laboratory (NEL) showed that the relative errors in the determination of water-cut ranged from $-1.49 \%$ to $48.8 \%$, which were deemed not satisfactory. Prafull et al. [19] proposed a sensing principle based on the open-ended microwave cavity resonator and near wall surface perturbation for non-intrusive measurement of water-cut, however, a homogeneously mixed liquid was required here due to the sensor only probing a small portion of the fluid near the pipe wall. Nyfors et al. [20] used a microwave resonator to measure the permittivity of the oil-water mixture in order to determine the water-cut, in the calculation that they proposed the permittivity of the oil and the water are required as inputs; with the water permittivity depending on the salinity and temperature.

These early application efforts produced promising results but also highlighted a number of areas requiring further study in order to understand the advantages and limitations of microwave sensors when used to monitor multiphase flow systems. The limitations/industrial drivers identified are related to: utilising the sensors during continuous flow and different flow regimes [13-15], the ability to accurately determine low water cuts [16], expansion of the measurement technique to non-homogenous systems [17], the large experimental errors resulting from certain configurations [18], the need to perform volumetric measurements [19] 
and the consideration of the influence of parameters such as temperature and salinity that can affect the measurements [20].

The majority of the previous studies in the field appear to have as a common denominator the sensitivity of microwave methods on the different flow regimes and thus the spatial phase distribution within a multiphase system. The present work will aim to investigate the interaction of the resonant electromagnetic field spatial distribution with multiphase systems in order to provide a better understanding in the practical implementation of the technique and potentially address some of the identified limitations.

\section{Materials and Methods}

\subsection{Materials}

Silicone oil with a viscosity of $5 \mathrm{cP}$, was purchased from Sigma-Aldrich and used as received.

\subsection{Resonant cavity and measurement system}

Microwaves are electromagnetic waves having a wavelength in the approximate range from $1 \mathrm{~mm}$ to $1 \mathrm{~m}$ and occupy the region between infrared and radio frequency wavelengths [21]. Consequently, an appropriately sized metallic cavity can be constructed, that confines the electromagnetic field in the microwave region of the spectrum and can resonate at these frequencies. When the cavity is loaded with a material under investigation, the resonance condition will be met at a lower frequency than for the unloaded cavity [22] depending on the load's complex permittivity. Therefore, a microwave resonator could be used to monitor changes in the resonant patterns introduced by a load consisting of a mixture of immiscible fluids. These changes can be correlated to the total complex permittivity of the fluid consisting of high (water) and a low permittivity (oil) phases. This means that when a pipe carrying wateroil mixture is inserted into the resonant cavity, the variation of the water volume fraction could be observed by the change of complex permittivity (dielectric constant and loss), which is indicated by frequency shift in the resonant pattern inside the cavity.

In the present study, a cylindrical microwave resonator with a hole at the centre to accommodate a pipe was employed (see Figure 1). This type of sensors has been commonly proposed in the past as suitable for measurements in multiphase systems [23]. The resonant cavity was made of copper and coupling to an external microwave source, Hewlett Packard 8753C network analyser utilising a 85047A S-parameter test set $(300 \mathrm{KHz}-6 \mathrm{GHz})$ (HewlettPackard, Palo Alto, California, USA), was achieved via two loop antennas. The diameters of cavity and central hole were $245 \mathrm{~mm}$ and $45 \mathrm{~mm}$ respectively and the height of the cavity was 24.7mm. The cavity and Vector Network Analyser (VNA) are shown in Figure 1. 


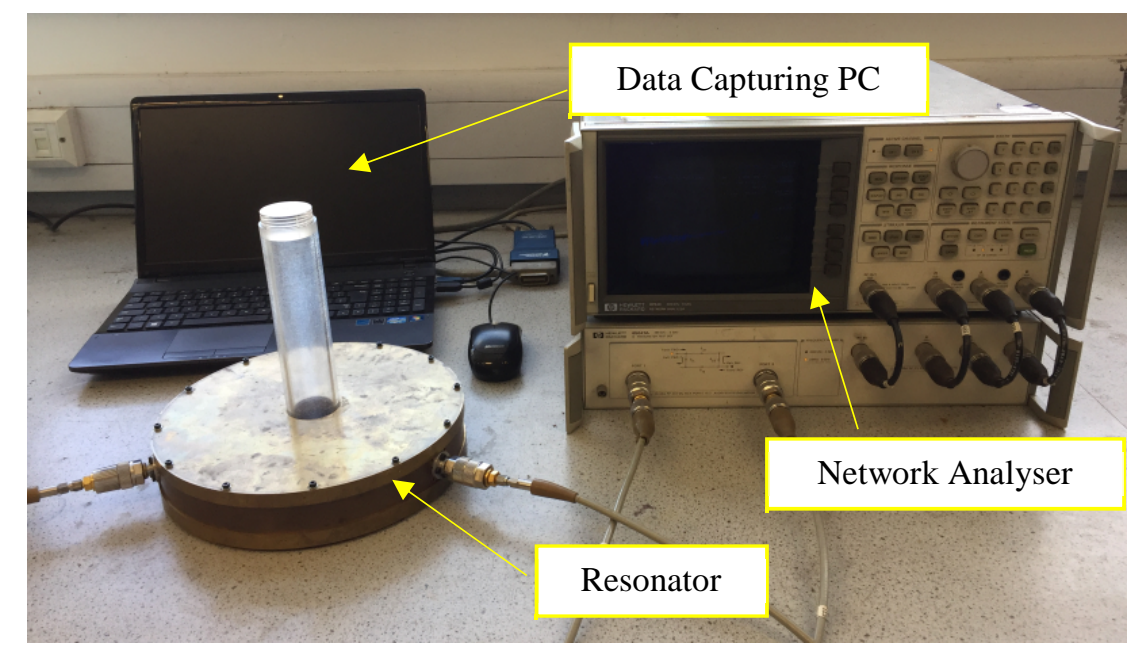

Fig.1 Benchtop Measurement system of a cylindrical resonant cavity

Various resonant modes like TM (transverse magnetic) and TE (transverse electronic) can exist simultaneously in the cavity resonator [24]. The resonant frequency for the TE and TM modes for the case of the unloaded/empty resonator are expressed mathematically as shown in Equations (1) and (2) respectively.

$$
\begin{aligned}
& \text { TE mode }: f_{n m l}=\frac{1}{2 \pi \sqrt{\mu \varepsilon}} \sqrt{\left(\frac{p_{n m}^{\prime}}{a}\right)^{2}+\left(\frac{l \pi}{d}\right)^{2}} \\
& \text { TM mode }: f_{n m l}=\frac{1}{2 \pi \sqrt{\mu \varepsilon}} \sqrt{\left(\frac{p_{n m}}{a}\right)^{2}+\left(\frac{l \pi}{d}\right)^{2}}
\end{aligned}
$$

Where $n, m$ and $l$ are the number of variations in the standing wave pattern along radial, circumferential and $\mathrm{z}$ directions of cavity. The permeability and the permittivity of the material are denoted by $\mu$ and $\varepsilon$ respectively. $a$ and $d$ are the internal radius and the height of the cylindrical resonant cavity. The $\mathrm{m}^{\text {th }}$ root of the Bessel function of the $\mathrm{n}^{\text {th }}$ order is given by $\mathrm{p}_{\mathrm{nm}}$ while $\mathrm{p}_{\mathrm{nm}}^{\prime}$ is the $\mathrm{m}^{\text {th }}$ root of the derivative of the Bessel function of the $\mathrm{n}^{\text {th }}$ order.

The experimental study focused on the $\mathrm{TM}_{010}$ and $\mathrm{TM}_{110}$ modes with resonant frequencies of the unloaded $\mathrm{TM}_{010}$ and $\mathrm{TM}_{110}$ modes are $937 \mathrm{MHz}$ and $1490 \mathrm{MHz}$ respectively as they were more practical from a measurement point of view. Therefore, two frequency ranges measured, one from $820 \mathrm{MHz}$ to $920 \mathrm{MHz}$ and the other from $1350 \mathrm{MHz}$ to $1450 \mathrm{MHz}$. The number of points was set to 401 and the power of the microwave source to $10 \mathrm{dBm}$. The IF bandwidth and averaging factor were adjusted to $100 \mathrm{~Hz}$ and 4 respectively.

The quality factor (Q-factor) is a dimensionless parameter that describes the energy balance within the resonator. The quality factor $\mathrm{Q}$ of a resonant cavity is defined as the ratio of the energy stored in the oscillating resonator to the energy dissipated per cycle by damping processes (Eq. (3)).

$$
Q \stackrel{\text { def }}{=} 2 \pi \times \frac{\text { energy stored }}{\text { energy dissipated per cycle }}
$$

The resonant frequency and the quality factor of each mode in the resonant cavity would change when fluids are flowing in a pipe placed through the centre cavity, due to the complex 
permittivity of the fluid. The scattering $S_{21}$ parameters, which is the ratio of output and input power, can be utilised to monitor the change in the frequency and Q-factor of each mode with the use of a VNA. The scattering $\left(S_{21}\right)$ parameters in the present experimental setup are related to the reflected and transmitted power to the microwave resonator as a function of frequency and have an amplitude and a phase. Typically, the amplitude is measured in decibels (dB) and the phase is measured in degrees. It must be emphasised that in the current investigation the dimensions and dielectric properties of the materials under test do not permit the use of perturbation techniques to accurately calculate the changes in frequency shift and Q-factor in an analytical way therefore a pure experimental approach has been followed instead.

\subsection{Continuous flow experiments}

Continuous flow experiments carried out on the liquid-liquid flow facility at the multiphase flow laboratory at the University of Nottingham. Silicone oil and normal tap water are the fluids used in the test. It is important to mention that the salinity of the water has not been altered for these tests. Fig. 2 displays the schematic diagram of this facility. It mainly consists of liquid supply systems, flowmeters, regulating valves, test sections and a separator. Silicon oil and water were supplied separately from two storage tanks, and the flow rates are then measured using electromagnetic flow meters immediately before the mixing unit. After the mixer where a fine dispersion is obtained, the oil-water mixture flows through a pipe (37 mm ID) that forms a $U$ bend as shown in the Fig. 3. This pipe section is then connected to a larger diameter pipe (127 mm ID) through a sudden expansion, which returns the liquid back to the separator. A resonant cavity with a Plexiglas tube inserted through its centre is placed at $1050 \mathrm{~mm}$ downstream of the bend in the upward arm of the $U$ bend (refer to Fig 3). A sampling valve is fitted at the lower end of the U section.

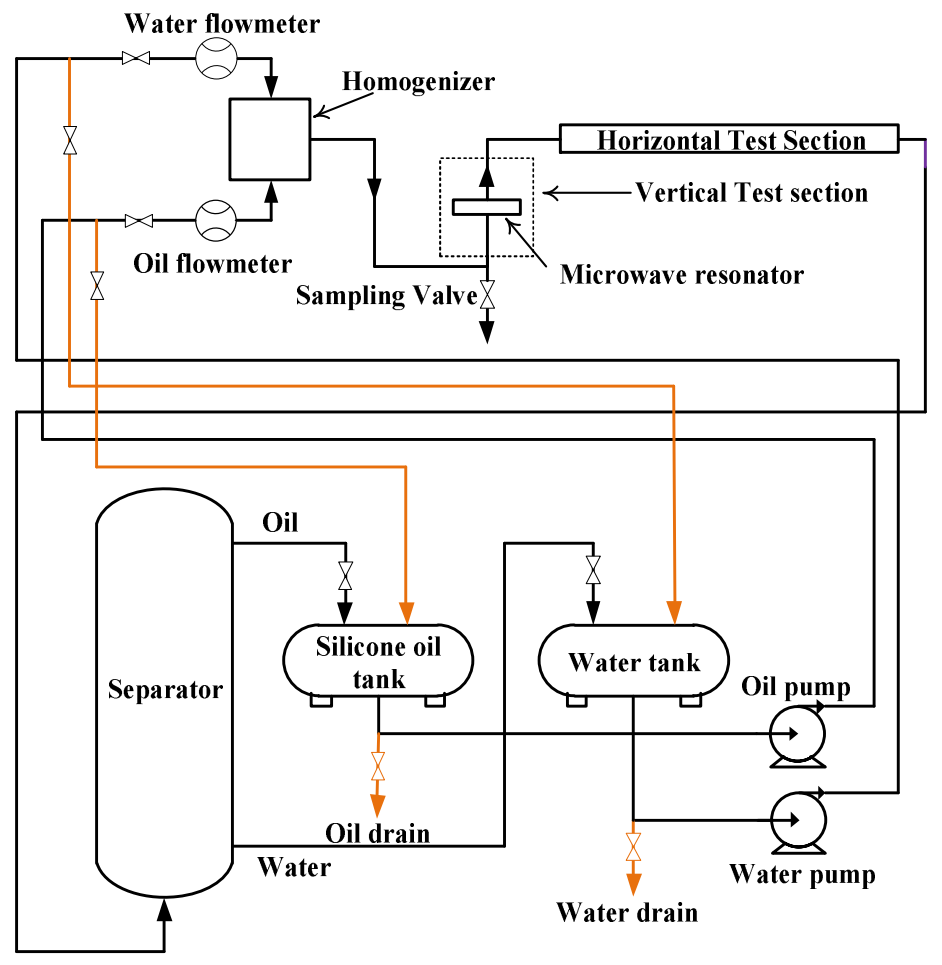

Fig.2 Schematic diagram of the liquid-liquid facility 


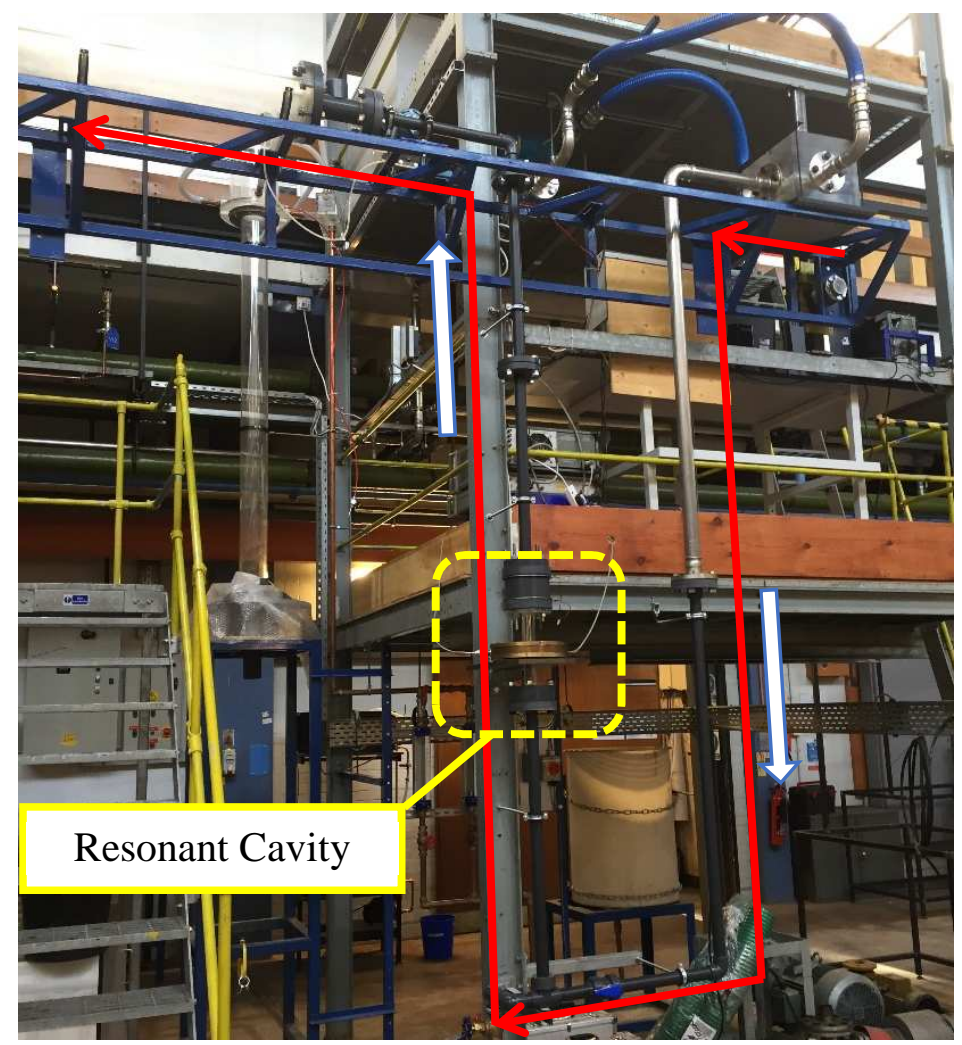

Fig.3 Resonant cavity mounted on the rig

The present study investigated oil flow rates of 50,75, 100, 125 and $150 \mathrm{~L} / \mathrm{min}$, while varying the water fractions in oil from $0 \%$ to $20.1 \%$ for each flow rate. To develop a calibration table, flowrates were set to known water cuts stated above and the frequency and the power associated with the peak measured once the steady state was achieved. A sample of the mixture is taken through the sampling port mounted near the resonator under flow conditions to establish the actual water volume fraction. This is necessary, as the local volumetric flow rate of each phase could be different to the inlet flowrates due to slip. The collected sample is allowed to settle and the volume fractions were established.

\subsection{Static (non-flow) experiments}

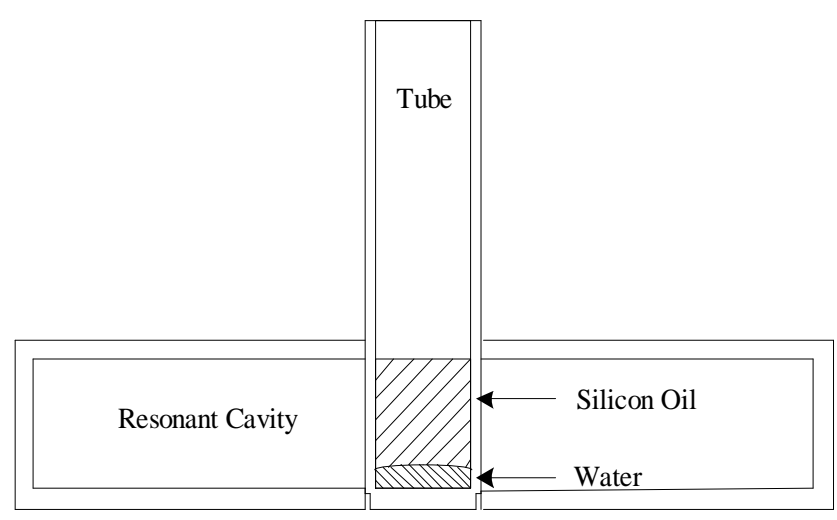

(a)

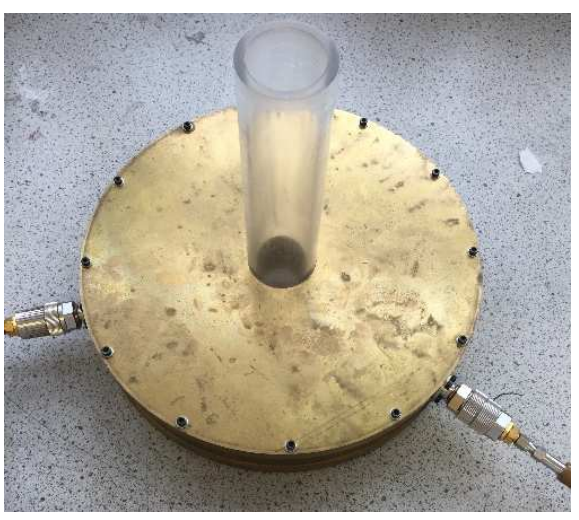

(b)

Fig.4 Arrangement of the static tests; (a) the schematic diagram showing a cross section through centreline (b) a photograph of the arrangement showing the resonance cavity and the pipe placement. 
In non-flow experiments (Figure 4), silicone oil and deionized water were used. The resonant cavity was laid flat on the table and the tube that holds the oil-water mixture placed vertically in the hole passes through the centre. In this this arrangement, silicon oil and the water would rest with the denser fluid (water) at the bottom. This experimental configuration was implemented in order to examine the performance of the proposed technique in flow situations where the water and oil phase are reasonably separated e.g. churn flow. Although, such flow conditions are regularly encountered in practice they are very difficult to be replicated in a controllable manner in our continuous flow experiments. Therefore, the inclusion of static (non-flow) experiments in the present study was deemed necessary.

In the static experiment, water-cut changed from $0 \%$ to $20.5 \%$, and all the experiments repeated three times to obtain the average values.

\section{Results and Discussion}

\subsection{Continuous flow experiments}

During the experiment, the resonant frequency of the partially filled cavity is the key measuring parameter. The resonant frequency of the partially filled cavity is determined directly by the peak observed in the $\mathrm{S}_{21}$ scattering parameter and the Q-factor is computed by the ratio of the resonant frequency over the bandwidth as determined by the $-3 \mathrm{~dB}$ points at either side of the resonant peak. Each measurement run took approximately $3 \mathrm{~s}$ and is the results were automatically logged with the use of a script written in Python. Figure 5 (a)-(b) display the resonant peaks of $\mathrm{TM}_{010}$ and $\mathrm{TM}_{110}$ modes with different water-cut.

According to the figures, as the water fraction increases, the resonant frequencies continuously shifted from approximately $890 \mathrm{MHz}$ to $860 \mathrm{MHz}$ for $\mathrm{TM}_{010}$ and $1.433 \mathrm{GHz}$ to $1.422 \mathrm{GHz}$ for $\mathrm{TM}_{110}$. This suggests that the frequency shift can be utilised to determine water-cut in oilwater flow through a careful calibration process.

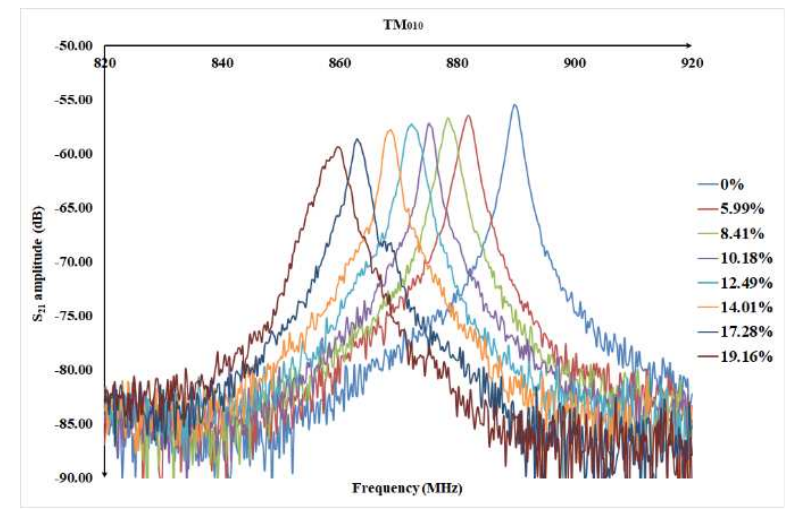

(a) $\mathrm{TM}_{010}, \mathrm{u}_{\mathrm{oil}}=125 \mathrm{~L} / \mathrm{min}$

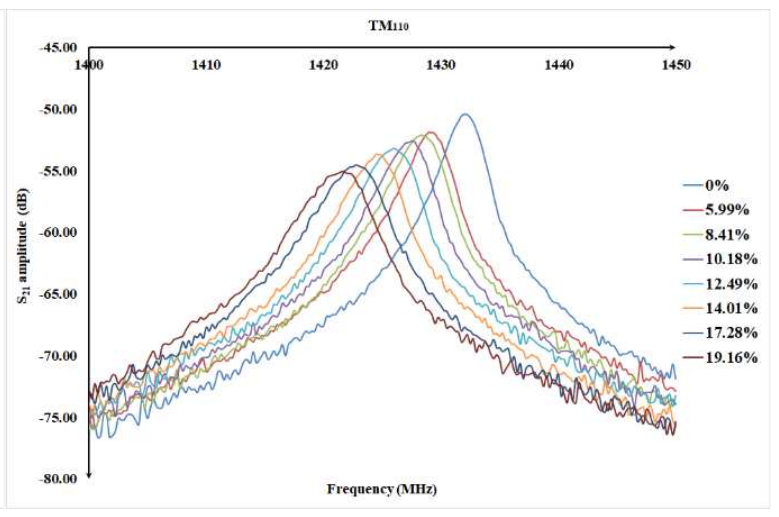

(b) $\mathrm{TM}_{110}, \mathrm{u}_{\mathrm{oil}}=125 \mathrm{~L} / \mathrm{min}$

Fig.5 Resonant peaks of different frequency and oil flow rates

In order to describe the relative frequency shift due to various water-cuts, a ratio is introduced as shown below.

$$
\eta=\frac{f-f_{0}}{f_{0}}
$$


This gives the relative shift in resonant frequency, where $f$ and $\mathrm{f}_{0}$ were the resonant frequency of oil-water mixture and $100 \%$ oil in the pipe respectively.

Figure 6 shows the $\eta$ versus water volume fraction of the liquid sample and the deviations of each points. All the data points in these two figures were the averaged values of three measurements. The largest standard deviation of each $\eta$ was 0.0011 .
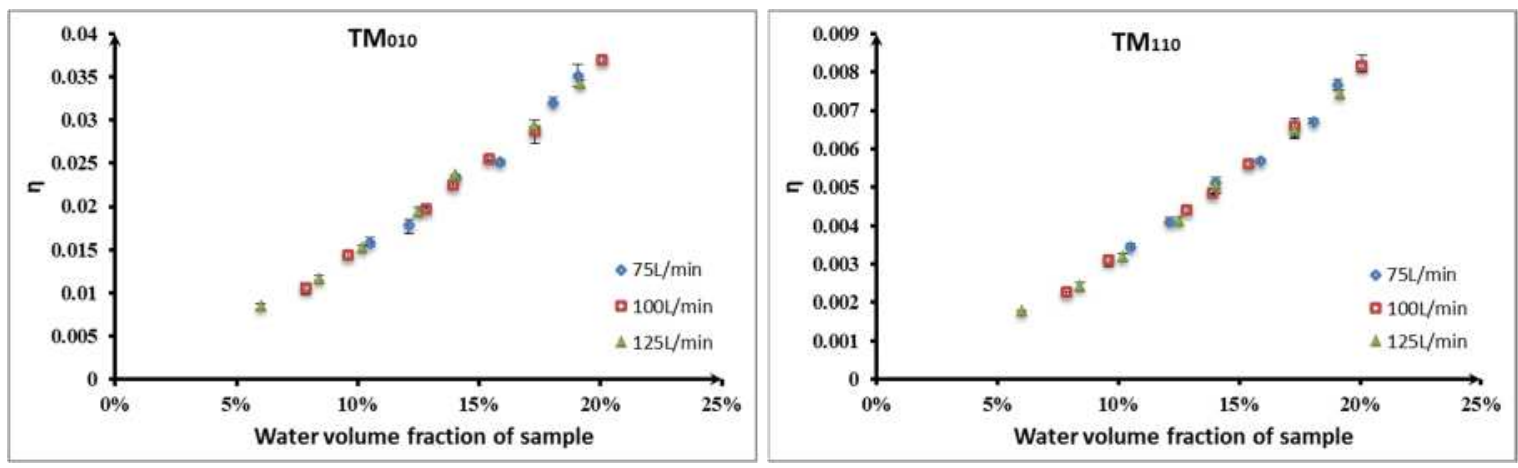

Fig.6 Relationship between $\eta$ and water volume fraction

Data points in figure 6 are overlapping for the flow rates considered indicating that the frequency shift is independent of the flow rate. The performance of resonant cavity only depends on the relative permittivity of the mixture, namely the water-cut of the oil-water flow.

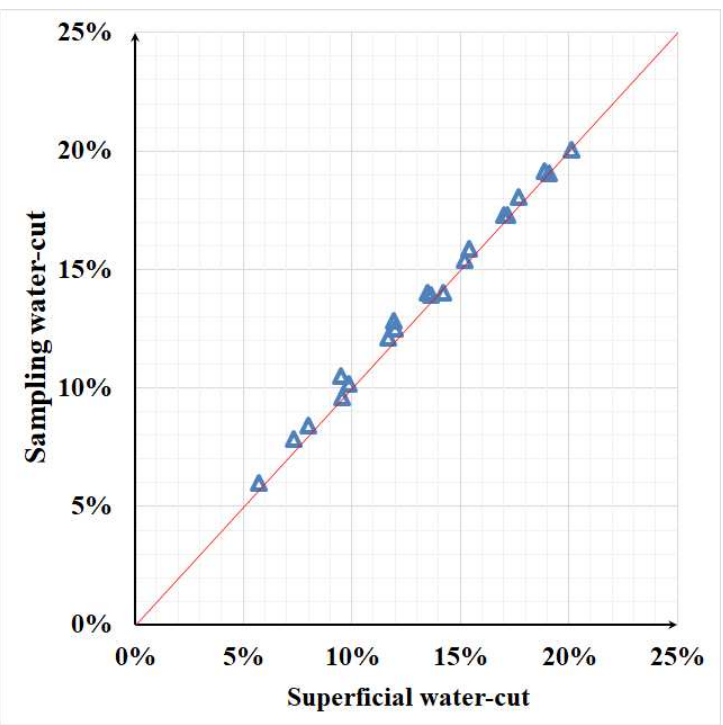

Fig.7 Comparison between sampling and superficial water-cut

Due to the difference in the velocities of oil and water, phase slip may exist in the two-phase upward flow and the water volume fraction measured using the microwave resonance cavity would be different from the values computed using the inlet flow rates or even from the sampling. In order to check whether the slip has affected the volume fraction measurement, the water-cut from the sampling and the water cut based on the inlet flowrate measurements were compared as shown in Figure 7. We can observe that the water-cuts determined via sampling are very close to the ones determined using the flow meter measurements. This indicates that the slip is negligibly small under the operating conditions of the present experiments. Since there was almost no slip in the experiment, water-cut of the sample could be used in the data analysis. 
In upward oil-water flow, variation of water-cut leads to different flow patterns like dispersed flow, froth flow and slug flow [25]. Froth flow and dispersed flow were occurred in the dynamic experiment, however, due to the limitation of the rig, slug flow was hard to form and we used static experiments to solve this issue.

\subsection{Static (non-flow) experiments}

The relationship between the resonant frequency and the amplitude of the $S_{21}$ parameter for the $\mathrm{TM}_{010}$ and $\mathrm{TM}_{110}$ modes is presented in Figure 8.

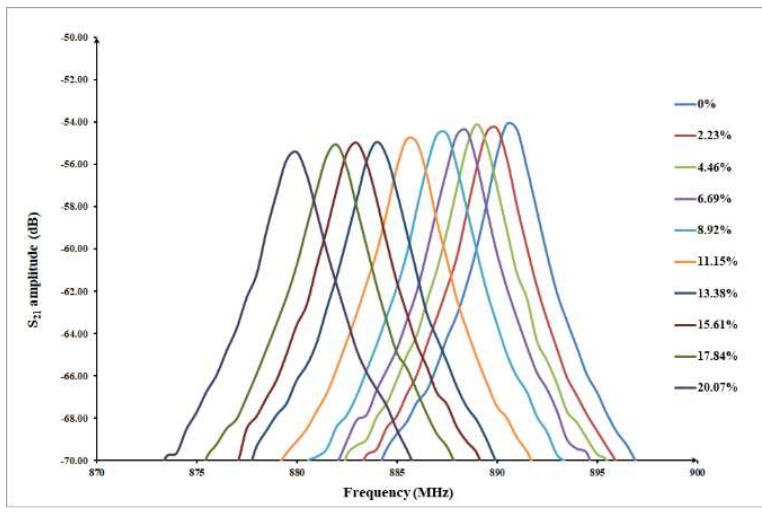

(a) $\mathrm{TM}_{010}$

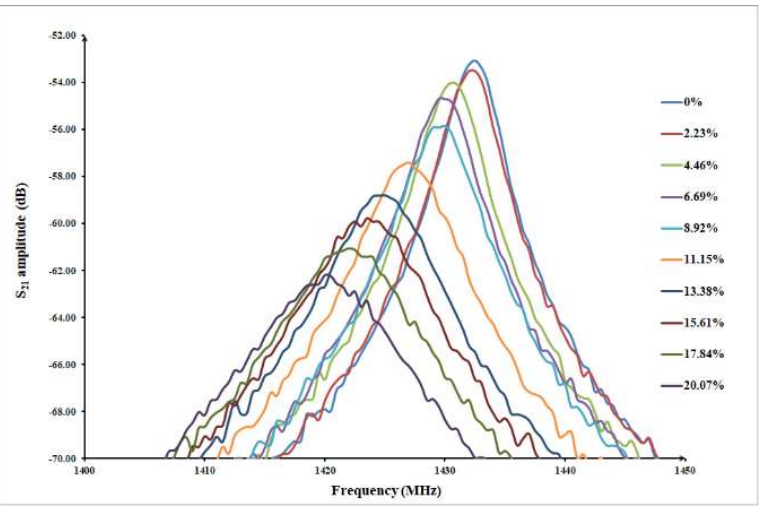

(b) $\mathrm{TM}_{110}$

Fig. $8 \mathrm{~S}_{21}$ amplitude versus frequency for the $\mathrm{TM}_{010}$ and $\mathrm{TM}_{110}$ modes. The legend in the graphs shows the different volumetric fractions of water in oil.

According to figure 8, for the $\mathrm{TM}_{010}$ mode, as the volumetric water fraction increases, the resonant frequencies shift from approximately $890 \mathrm{MHz}$ to $880 \mathrm{MHz}$ and the amplitude of the resonant peaks decreases by approximately $1 \mathrm{~dB}$. For the $\mathrm{TM}_{110}$ mode, the resonant frequencies shift from approximately $1.433 \mathrm{GHz}$ to $1.42 \mathrm{GHz}$ and the amplitude of the resonant peaks decreases by approximately $9 \mathrm{~dB}$ for the highest increase in the volumetric water fraction. The experimental results imply that the variation of the volumetric water fraction in the static oilwater mixture could be described by the frequency shift and the amplitude decrease. In the latter, $\mathrm{TM}_{110}$ mode produces larger changes than $\mathrm{TM}_{010}$ for different volumetric water concentrations. Figure 9 shows the change in the Q-factor versus water cut for the two resonant modes. $\mathrm{TM}_{110}$ exhibits a substantial change in Q-factor in comparison to the $\mathrm{TM}_{010}$ mode, in the $\mathrm{TM}_{110}$ mode. 


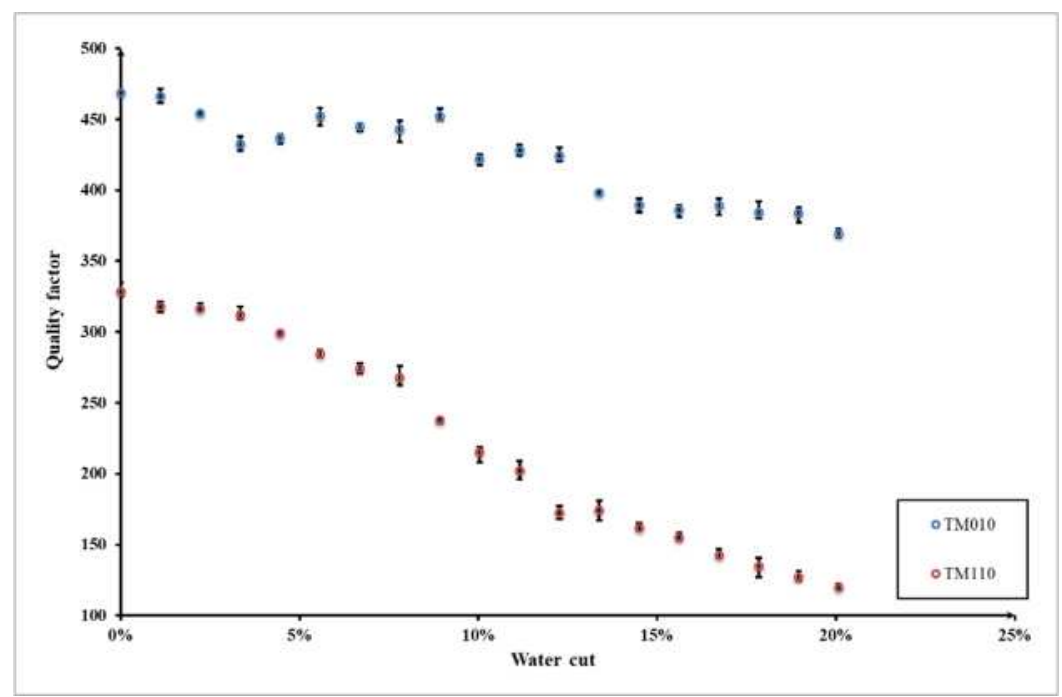

Fig.9 Quality factor of $\mathrm{TM}_{010}$ and $\mathrm{TM}_{110}$ modes

Similarly, to the continuous flow experiments, $\eta$ was also quantified. Three sets of data were collected and analysed, to verify the repeatability of the experiment. Figure 10 gives the average value of $\eta$ with the corresponding standard deviations shown as error bars, the biggest value of standard deviation observed was 0.0009 .

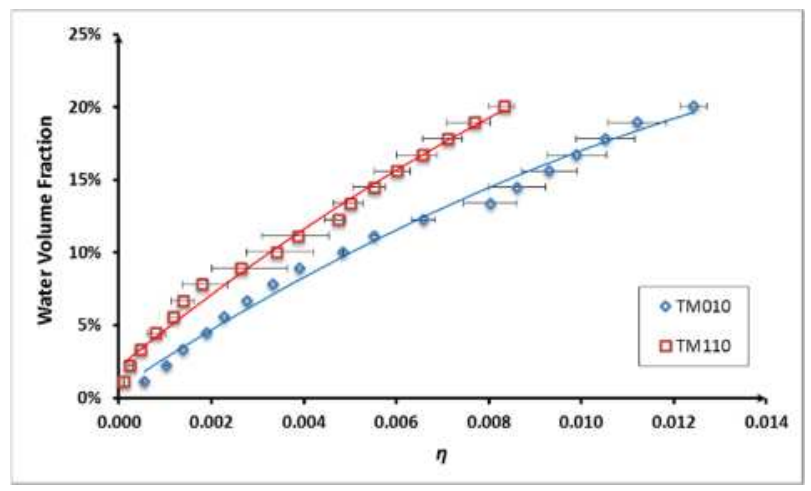

Fig.10 $\eta$ and water volume fraction in the static experiment

As expected, the water phase distributions between the continuous flow and static experiments are significantly different, and this appears to have a significant impact on the behaviour of $\mathrm{TM}_{010}$ and $\mathrm{TM}_{110}$ modes. Figure 11 shows the results of static and dynamic experiments for both modes and for a range of flowrates $(50 \mathrm{~L} / \mathrm{min}-150 \mathrm{~L} / \mathrm{min})$. From the figure, we can see that for the $\mathrm{TM}_{010}$ mode (which exhibits the highest relative frequency shift), the $\eta$ values of the static experiment were lower than that of continuous flow test with the same water-cut. On the contrary, in the $\mathrm{TM}_{110}$ mode (associated with much lower relative frequency shift), $\eta$ exhibits better agreement between the static and continuous flow experiments. The oil flowrates appear not to have a substantial impact in in the performance of either resonant mode. 


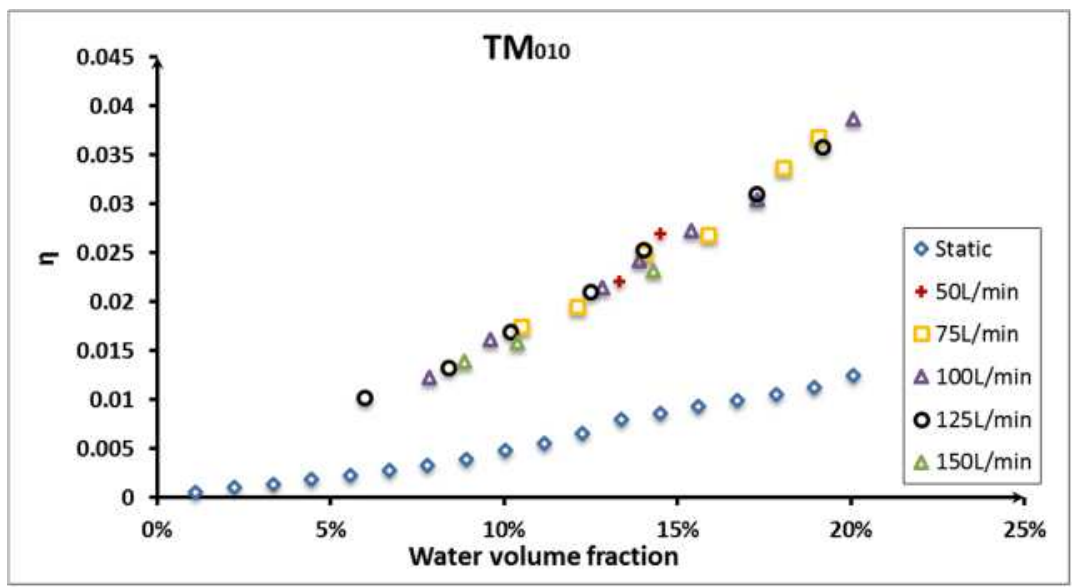

(a) $\mathrm{TM}_{010}$ mode

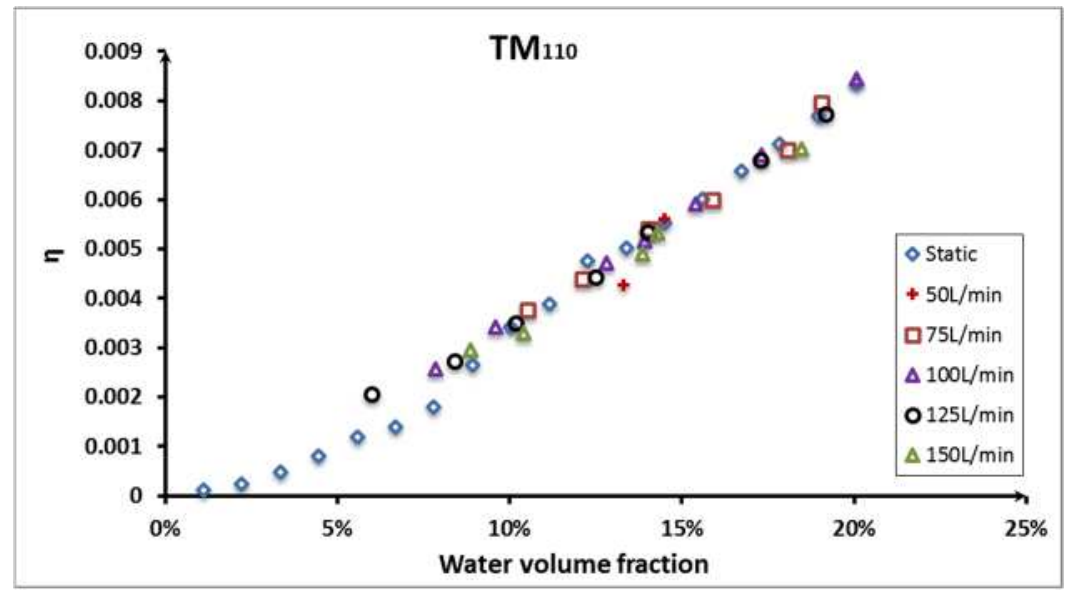

(b) $\mathrm{TM}_{110}$ mode

Fig. 11 Comparison between static and dynamic experiments

These observations potentially could be attributed to the different electric field spatial distribution patterns of two resonant modes.

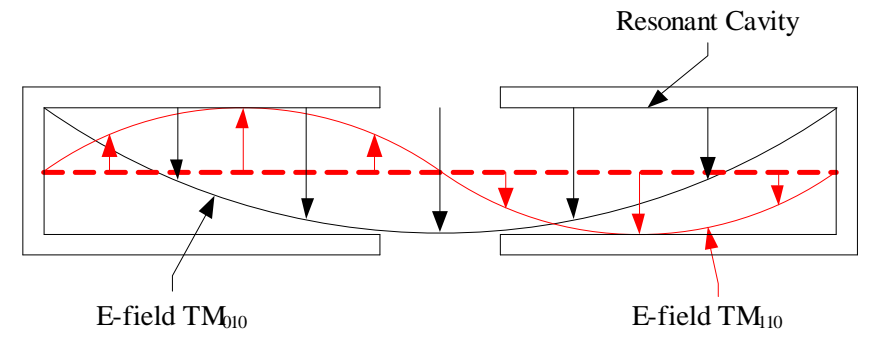

Fig.12 Electric fields of $\mathrm{TM}_{010}$ and $\mathrm{TM}_{110}$ modes in unloaded cavity

Figure 12 shows the electric fields of $\mathrm{TM}_{010}$ (black line) and $\mathrm{TM}_{110}$ (red line) modes in unloaded cavity. The placement of the tube and oil-water mixture would typically reduce the intensity of the field and the resonant frequency. In $\mathrm{TM}_{010}$ mode, the electric field is at its maximum intensity at the axis of the cavity when empty [11]. Whereas for $\mathrm{TM}_{110}$ mode, there are two maximum electric field peaks located inside the cavity and at the location of the tube the field intensity is at a minimum. Therefore, the $\mathrm{TM}_{010}$ is expected to exhibit a stronger coupling when the high permittivity phase (water) is confined near the entry point of the tube to the cavity 
(static experiment) as opposed to when the same volume of water is evenly dispersed in the pipe inside the cavity which explains the experimental observation in Figure 11 a. In an analogous approach since the electric field for the $\mathrm{TM}_{110}$ mode at the location of the sample is at a minimum the coupling is less affected by the spatial distribution of the high permittivity water phase which explains the agreement in the experimental results between the static and continuous flow experiments (Figure 11(b)). These observations are further supported by the data in Figure 9 and 13 where one can see that the $\mathrm{TM}_{010}$ exhibits significantly lower Q-values during the continuous flow experiment when compared to the static experiment which indicates a reduced level of interaction when the water phase is confined near bottom of the cavity. The experimental observations suggest that the performance of the $\mathrm{TM}_{110}$ mode is not affected to the same extend by the different flow regimes, making that mode more suitable for the determination of water-cut in the oil-water mixture.

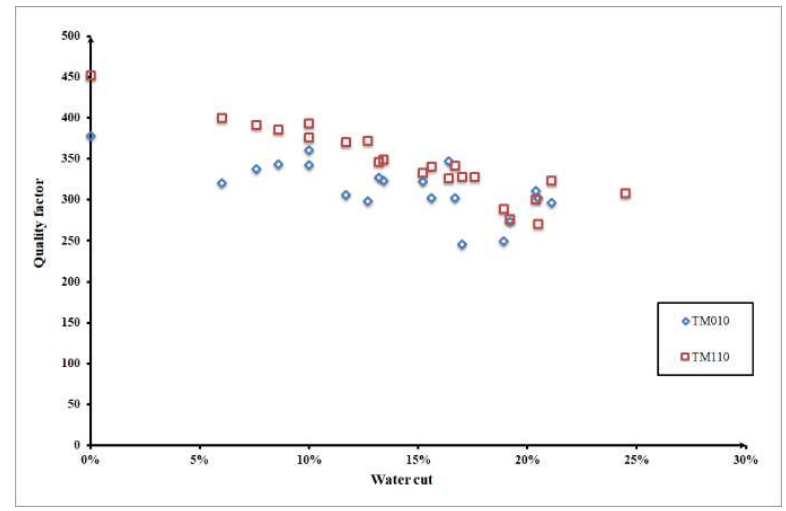

Fig.13 Effect of water-cut on the quality factor of $\mathrm{TM}_{010}$ and $\mathrm{TM}_{110}$ modes in dynamic experiment

In order to establish a correlation between water-cut and $\eta$, the complex permittivity $\varepsilon^{*}$ of the oil and water phases is a key variable. In the oil-water mixture, $\varepsilon^{*}$ of the mixture is dependent on the volume fraction and $\varepsilon^{*}$ of the respective constituent phases [26]. A widely utilised relationship that describes $\varepsilon^{*}$ water-in-oil emulsions has been proposed by Brugeman [27] (Eq. (5)) and has been extensively verified in the literature [26, 28].

$$
\frac{\varepsilon_{w}^{*}-\varepsilon_{m i x}^{*}}{\varepsilon_{w}^{*}-\varepsilon_{o}^{*}}\left(\frac{\varepsilon_{o}^{*}}{\varepsilon_{m i x}^{*}}\right)^{\frac{1}{3}}=1-\theta
$$

Where $\varepsilon^{*}, \varepsilon^{*}{ }_{w}, \varepsilon^{*}$ mix are the oil, water and mixture permittivities respectively and $\theta$ is the water-cut. For a given temperature and frequency, $\varepsilon^{*}{ }_{o}$ and $\varepsilon^{*}{ }_{w}$ are constants, so Eq. (5) is transformed to :

$$
\theta=k_{1} \cdot \varepsilon_{\text {mix }}^{* \frac{2}{3}}+k_{2} \cdot \varepsilon_{\text {mix }}^{*-\frac{1}{3}}+k_{3}
$$

Which expresses the relationship between water-cut and $\varepsilon_{m i x}$. Where $k_{1}, k_{2}$ and $k_{3}$ are constants.

The relative frequency shift in the resonator, $\eta$, linked directly to $\varepsilon^{*}{ }_{m i x}$ and in certain systems, such as the perturbation of a pill-box cavity by a dielectric rod [29] and the sample inserted along the axis of a cylindrical cavity [30]. These researches give a common conclusion that $\eta$ is a first-order function of $\varepsilon^{*}{ }_{m i x}$, that is 


$$
\eta=f\left(\varepsilon_{m i x}^{*}\right)
$$

Combining Eq. (6) and (7), a correlation (Eq. (8)) could be established with some coefficients need to be determined.

$$
\theta=a \cdot \eta^{\frac{2}{3}}+b \cdot \eta^{-\frac{1}{3}}+c
$$

where $\mathrm{a}, \mathrm{b}$ and $\mathrm{c}$ were obtained from experimental data of the static case for the $\mathrm{TM}_{110}$ mode.

$$
\theta=4.9817 \cdot \eta^{\frac{2}{3}}+0.0012 \cdot \eta^{-\frac{1}{3}}-0.0141
$$

Fig. 14 shows the accuracy if the fit of Eq. (9) in both the static and dynamic experiment.

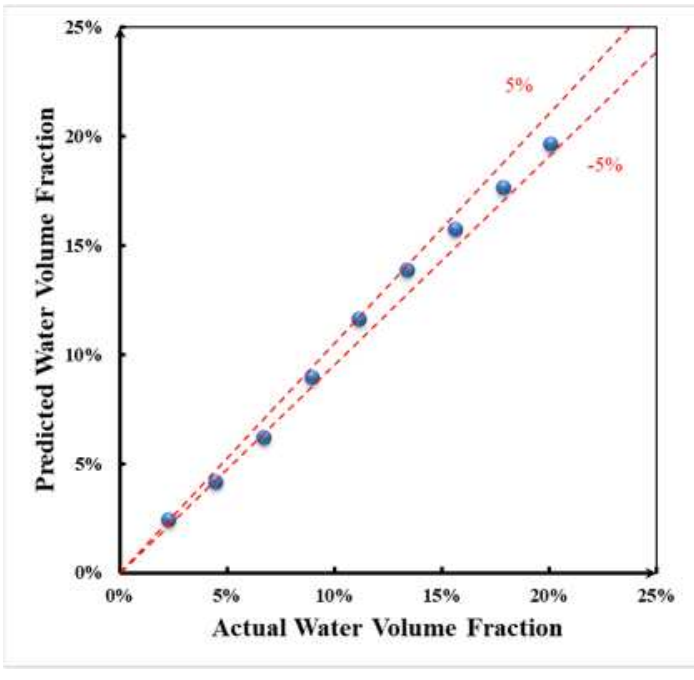

(a) Static experiment

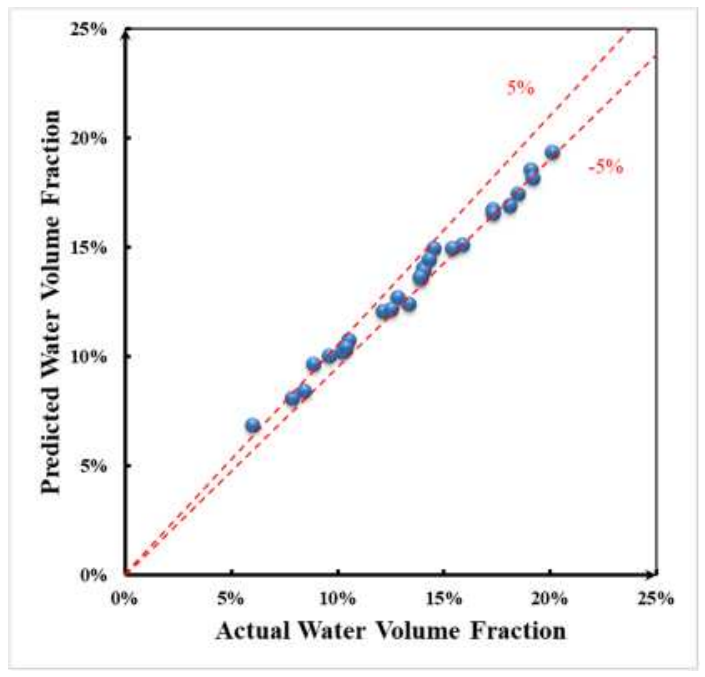

(b) Dynamic experimental

Fig. 14 Predicted water volume fraction using Eq. (9) plotted against the measured volume fraction for the a) static and b) dynamic experiment.

In Fig. 14, y-axis indicates the different water-cut values predicted by Eq. (9) and x-axis shows the actual water-cut values as determined by the oil and water flowmeters. From Fig. 14(a) we can see the relative errors between the fitted and actual water-cut in the static experiment ranged from $-6.94 \%$ to $4.5 \%$ except the minimum water-cut $(2.23 \%)$ with an estimated value of $2.44 \%$ (absolute error was $0.21 \%$ ).

Applying Eq. (9) to the dynamic experimental data, the relative errors between the predicted and actual water-cut were from $-6.53 \%$ to $9.16 \%$ except the minimum water-cut (5.99\%) with an estimated value of $6.86 \%$ (absolute error was $0.87 \%$ ). Approximately $78 \%$ of the total data points were inside the $-5 \%$ to $5 \%$ relative error boundary. The results suggest that for the $\mathrm{TM}_{110}$ mode, data from a single flow rate can be used to calculate $n$ and predict the water-cut at different flow rates.

\section{Conclusions}

In the present study a microwave resonator of cylindrical geometry was used to determine water cut under stratification and continuous flow of an oil/water system in a non-invasive manner. The study focused on water cuts below $20 \%$, which also are the more relevant scenario 
in real applications and flowrates varying between 5-20 L/min. Two resonant modes $\mathrm{TM}_{010}$ $\mathrm{TM}_{110}$ where selected to examine how the electric field spatial distribution within the resonator affects the accuracy and experimental performance of the system when characterising multiphase flows. It was found that the $\mathrm{TM}_{110}$ mode of cylindrical microwave resonator is less dependent on the phase distribution of the multiphase system and can provide accurate results irrespectively of the flow rate and flow pattern. The relative errors between the predicted and actual water-cut were from $-6.53 \%$ to $9.16 \%$, and relative errors of $77.78 \%$ of the total data points were inside $-5 \%$ to $5 \%$.

The superior performance of the $\mathrm{TM}_{110}$ mode, when is used to monitor multiphase system, is attributed to the presence of an electric field minimum at the location of the tube which ensures a consistent (albeit weaker) coupling throughout the different flow regimes compared to the $\mathrm{TM}_{010}$ mode which although is more sensitive, appears to be affected more by the flow-pattern under consideration. The results of the present study indicate that by careful selection of the microwave sensor's geometry and frequency the technique can be applied successfully to characterise multiphase systems in an accurate and non-intrusive manner and are capable to overcome limitations that historically are associated with such systems.

The potential applications of the proposed measuring configuration could be in the field of oil and gas engineering, where it could be used to monitor the water quantity in petroleum output. Combined with some economic flowmeters (e.g. vortex, turbine or differential pressure), can form an online multiphase flowmeter, which can be used to optimise the operation and performance of large and costly separators typically utilised in such processes.

\section{Acknowledgements}

This work was financially supported by the International Postdoctoral Exchange Fellowship Program (No. 20160049) by the Office of China Postdoctoral Council.

\section{Reference}

1. Strizzolo, C.N. and J. Converti, Capacitance sensors for measurement of phase volume fraction in two-phase pipelines. IEEE Transactions on instrumentation and measurement, 1993. 42(3): p. 726-729.

2. Luggar, R., et al., Energy dispersive X-ray scatter for measurement of oil/water ratios. Nuclear Instruments and Methods in Physics Research Section A: Accelerators, Spectrometers, Detectors and Associated Equipment, 1999. 422(1-3): p. 938-941.

3. Roshani, G., et al., Precise volume fraction prediction in oil-water-gas multiphase flows by means of gamma-ray attenuation and artificial neural networks using one detector. Measurement, 2014. 51: p. 34-41.

4. Jaworski, A.J. and T. Dyakowski, Measurements of oil-water separation dynamics in primary separation systems using distributed capacitance sensors. Flow Measurement and Instrumentation, 2005. 16(2-3): p. 113-127.

5. Da Silva, M., et al., Phase fraction distribution measurement of oil-water flow using a capacitance wire-mesh sensor. Measurement Science and Technology, 2011. 22(10): p. 104020.

6. Boyer, C., A.-M. Duquenne, and G. Wild, Measuring techniques in gas-liquid and gasliquid-solid reactors. Chemical Engineering Science, 2002. 57(16): p. 3185-3215.

7. Tompkins, C., H.-M. Prasser, and M. Corradini, Wire-mesh sensors: A review of methods and uncertainty in multiphase flows relative to other measurement techniques. Nuclear Engineering and Design, 2018. 337: p. 205-220. 
8. Thorn, R., G.A. Johansen, and B. Hjertaker, Three-phase flow measurement in the petroleum industry. Measurement Science and Technology, 2012. 24(1): p. 012003.

9. Bernou, C., D. Rebière, and J. Pistré, Microwave sensors: a new sensing principle. Application to humidity detection. Sensors and Actuators B: Chemical, 2000. 68(1): p. 88-93.

10. Nyfors, E., Industrial microwave sensors -A review. Subsurface Sensing Technologies and Applications, 2000. 1(1): p. 23-43.

11. Mehdizadeh, M., Microwave/RF applicators and probes: for material heating, sensing, and plasma generation. 2015: William Andrew.

12. Thorn, R., G. Johansen, and E. Hammer, Recent developments in three-phase flow measurement. Measurement Science and Technology, 1997. 8(7): p. 691.

13. Yang, Y., B. Scott, and B. Cregger. The design, development, and field testing of a water-cut meter based on a microwave technique. in SPE Annual Technical Conference and Exhibition. 1990. Society of Petroleum Engineers.

14. Ávila, H.E., D.J. Pagano, and F. Rangel de Sousa, Water fraction measurement using a $R F$ resonant cavity sensor. Instrumentation viewpoint, 2013(14): p. 12-12.

15. Oon, C.S., et al., Experimental study on a feasibility of using electromagnetic wave cylindrical cavity sensor to monitor the percentage of water fraction in a two phase system. Sensors and Actuators A: Physical, 2016. 245: p. 140-149.

16. Ashton, S., et al. Development and trial of microwave techniques for measurement of multiphase flow of oil, water and gas. in SPE Asia Pacific Oil and Gas Conference. 1994. Society of Petroleum Engineers.

17. Hogan, B., A. Al-Shamma a, and J. Lucas. Real-time multiphase metering using nonintrusive microwave sensor. in BHR Group Conference Series Publication. 2000. Bury St. Edmunds; Professional Engineering Publishing; 1998.

18. Wylie, S.R., A. Shaw, and A.I. Al-Shamma'a, RF sensor for multiphase flow measurement through an oil pipeline. Measurement Science and Technology, 2006. 17(8): p. 2141-2149.

19. Sharma, P., L. Lao, and G. Falcone, A microwave cavity resonator sensor for water-inoil measurements. Sensors and Actuators B: Chemical, 2018. 262: p. 200-210.

20. Nyfors, E.G. and A. Wee. Measurement of mixtures of oil, water, and gas with microwave sensors: new developments and field experience of the MFI MultiPhase and WaterCut meters of Roxar. in Subsurface Sensing Technologies and Applications II. 2000. International Society for Optics and Photonics.

21. Berube, M., The American Heritage Dictionary: Second College Edition. 1985: Houghton Mifflin.

22. Nyfors, E.G., Cylindrical microwave resonator sensors for measuring materials under flow. 2000: Helsinki University of Technology.

23. Falcone, G., G. Hewitt, and C. Alimonti, Multiphase flow metering: principles and applications. Vol. 54. 2009: Elsevier.

24. Maab, H. and Q.A. Naqvi, Fractional rectangular cavity resonator. Progress In Electromagnetics Research, 2008. 9: p. 69-82.

25. Govier, G.W., G.A. Sullivan, and R.K. Wood, The upward vertical flow of oil - water mixtures. The Canadian Journal of Chemical Engineering, 1961. 39(2): p. 67-75.

26. Sherman, P., Emulsion science. 1968: Academic Press.

27. Bruggeman, V.D., Berechnung verschiedener physikalischer Konstanten von heterogenen Substanzen. I. Dielektrizitätskonstanten und Leiffähigkeiten der Mischkörper aus isotropen Substanzen. Annalen der physik, 1935. 416(7): p. 636-664.

28. Banhegyi, G., Numerical analysis of complex dielectric mixture formulae. Colloid and Polymer Science, 1988. 266(1): p. 11-28. 
29. Carter, R.G., Accuracy of microwave cavity perturbation measurements. IEEE Transactions on Microwave theory and techniques, 2001. 49(5): p. 918-923.

30. Lakshminarayana, M., L. Partain, and W. Cook, Simple microwave technique for independent measurement of sample size and dielectric constant with results for a Gunn oscillator system. IEEE Transactions on Microwave Theory and Techniques, 1979. 27(7): p. 661-665. 